\title{
Automatic Threading and Metal Cutting in Smart Industries Using IoT
}

\author{
Brindha $\mathrm{J}^{\mathrm{a}, 1}$, Dharshan $\mathrm{H}^{\mathrm{b}}$, Maheshwar $\mathrm{P}^{\mathrm{b}}$ and Mugilan $\mathrm{SB}^{\mathrm{b}}$ \\ ${ }^{a}$ Assistant Professor, Dept of E\&I Panimalar Engineering College, Chennai, India \\ ${ }^{b}$ UG Scholar, Dept of E\&I, Panimalar Engineering College, Chennai, India
}

\begin{abstract}
The key objective of the project is to automate an industrial process of threading and metal cutting. The main objective of the project is that it uses IOT in industries with Artificial Intelligence to monitor and control the industrial processes thus increasing productivity and to reduce cost and man power. The system uses a microcontroller for processing all user commands and EEPROM chip to save data. Digital voltmeter, ammeter, wattmeter and IR cam etc., can be linked to the same IOT device without any additional circuits.
\end{abstract}

Keywords. IOT, RFID, WIFI module.

\section{Introduction}

In recent years industrial IOT applications have been developed and implemented effectively. It begins with Radio Frequency Identification (RFID) technology, which allows microchips to transmit the identification information through wireless communication to a reader. With the help of RFID readers, people track and monitor automatically using RFID tags. Wireless sensor networks (WSN) helps to interconnect the suitable sensors to sense and monitor the processes like environmental monitoring, industrial monitoring and traffic monitoring. The upcoming technology is the combination of Industrial Internet Of Things (IIOT) with Artificial Intelligence (AI). IIOT is the best way of connecting industrial hardware and sensors, to collect information from these connected devices to process data in a useful way by internet. ${ }^{[2]}$ IOT supports data accession, collection, interpretation, and visualization. IOT includes computers, intelligent devices, wired and wireless communication and cloud computing. RF (radio frequency) and Bluetooth technologies are used to control and monitor the industrial applications manually but the major drawback is, it cannot cover wide range and operator should be in that particular range. In this proposed paper short distance communication using IOT is carried out in industrial environment from anywhere in the world.

\section{Existing Methods}

In the existing system, all machines are operated by person and at the same time it is monitored by them. All meter readings are recorded in manual method.In industries, traditional method is followed to count the number of production rate as they are pasting bar code in every product and the bar code is scanned to count the number of products produced per day, by doing this, the production rate is viewed.

\footnotetext{
${ }^{1}$ Brindha J Assistant Professor, Department of E\&I, Panimalar Engineering College, Chennai, India.

Email id: brindha.balajisrivatsan@gmail.com
} 


\section{Proposed System}

A mobile app is developed to control and monitor. This app contains many modules namely OPS (Overload Protection Services), PCR (Power Consumption Recorder), Timer, Scheduler, etc.[1] This mobile app is connected to the microcontroller board over internet. Through GUI (Graphical User Interface) the program in microcontroller is changed for real time status. And thus, the modem decodes information and transfers it to the microcontroller for further processing. These relays operate in a range of 10A-15A. Here, machines are designed to run on specific RPM (Rotation Per Minute) with $+/-30 \mathrm{~V}$ from $220 \mathrm{~V}$. So small change in volt up to 30 has no effect.

\subsection{Major Units}

\subsubsection{Distribution Unit}

The work piece which are to be supplied to different units are collectively available in this units. The work piece is supplied to the respective unit through conveyor belt. The iron \& steel plant in the form of metal sheets with size ranging from $5 \mathrm{~mm}$ to $10 \mathrm{~mm}$ as lot depending upon requirements or Car Company.

\subsubsection{Metal Cutting Unit}

The metal sheets are cut in required size using metal cutter machine then the corners are grinded for smooth finishing. Door is made in which a multitude of panels each is split in two or three parts and braces will be either welded or bolted.

\subsubsection{Specifications}

1. Supply Input: $230 \mathrm{~V}$ AC

2. Load: up to $500 \mathrm{~W}$

\subsubsection{Threading Unit}

As assembly area is the threading unit of workers to assemble all the instrumentation materials like interior lights, door and trim panels, wiring systems, seats, and glasses where the threading takes place.

\subsubsection{Testing Unit}

In this way the assembly plants can predicts that the products arriving at their receiving ends are Statistical Process Control approved. In advanced assembly plants has small RF transponder that is attached to the floor pan and chassis.

\subsection{Working of Proposed System}

Here in proposed system[2] the metal cutting and threading unit is controlled and monitored using IOT. As the mobile app is interfaced with the loads any input can be given in the mobile app itself. The input is received by the Wi-Fi antenna in the microcontroller board then it is sent as a command to microcontroller. 


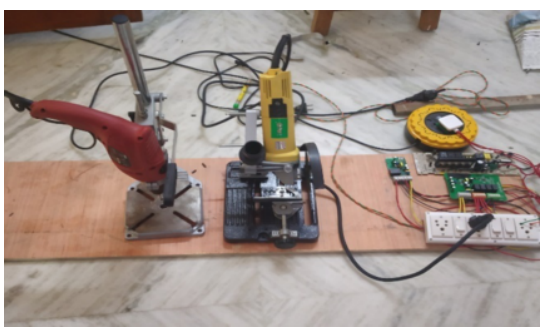

Figure 1. Working of proposed system

After producing output, the output data is transmitted to memory through the Wi-Fi antenna of microcontroller board. Then, this output data is received and shown in the display of mobile app.

\subsection{System Framework}

The Amazon AWS is used to store or send data; the user can operate from any place since cloud server is used. Through LTE modem the data is connected to the router which in turn connected to the Wi-Fi module. An IP (Internet Protocol) address or FQDN (Fully Qualified Domain Name), The biggest advantage of this system is the user can operate from any location. If the user is near the load, local intranet can be used. If the user is far away from the load, cloud server can be used to operate the loads.

\subsection{Number of Input /Output}

- Input: Input Single input power supply of $230 \mathrm{~V}$

- $\quad$ Output: one output from threading machine controller another from metal b cutting machine controller.

\subsection{Factors to be controlled}

1. Power

2. Voltage

3. Energy

\section{Software Description}

\subsection{PCR Module}

In PCR module the recorded data can be imported to android mobiles over Wi-Fi network/4G. The data will be saved to download folder in .csv file which can be opened using MS excel for detailed analysis. 


\subsection{OPS Module}

Maximum current, maximum power, minimum power values can be set here to protect the loads. OPS module executes itsfunction before operating the relays. So, the relays switch according to the set value.

\section{Hardware Description}

\subsection{IOT Main Board}

In the IOT main board we have use the model of 6F5CN74639.

\subsection{Metal Cutting Machine Controller}

This circuit controls the output of the AC motor linked to[2] the IOT device. Once user command is received from App signal is sent to the microcontroller and motor start rotating.

\subsection{Screw threading machine controller}

This circuit has two parts. One will control High Torque $12 \mathrm{v}$ Dc motors and the second will control AC motor linked to the IOT device.

\subsection{LM324N Circuit}

LM324N operates on a single power supply and with wide range of voltages. The amplifier goes to ground and it also allows direct sensing ground. LM324N is suitable with all forms of logic circuits.

\subsection{CEP50N06}

Zit is an N-Channel HEXFET Power MOSFET that comes in a TO-220AB package and operates on $55 \mathrm{~V}$ and $110 \mathrm{~A}$. Here in this project IRF3205 is used since it falls under the category of ultra-LOW on-resistance. Fast switching rate and operating temperature around $175^{\circ} \mathrm{C}$.

\section{Results and Discussion}

Table 1. Result Value

\begin{tabular}{|l|l|l|l|}
\hline Factor & $\begin{array}{l}\text { Expected } \\
\text { Output }\end{array}$ & Existing & Proposed \\
\hline Threading & $5 \mathrm{~mm}$ & $\begin{array}{l}5 \mathrm{~mm}+/ \\
1 \mathrm{~mm}\end{array}$ & $5 \mathrm{~mm}$ \\
\hline Voltage & $\begin{array}{l}\text { Auto off } \\
\text { if }>250\end{array}$ & $240+/$. & $240-250$ \\
\hline Total & $\begin{array}{l}\text { Auto off } \\
\text { Load }\end{array}$ & $2500-\gamma$ & 2500 \\
& if & 1000 & \\
& P>2500 & & \\
\hline
\end{tabular}

From the table we come to know that the expected value in order to increase the efficiency is achieved. 


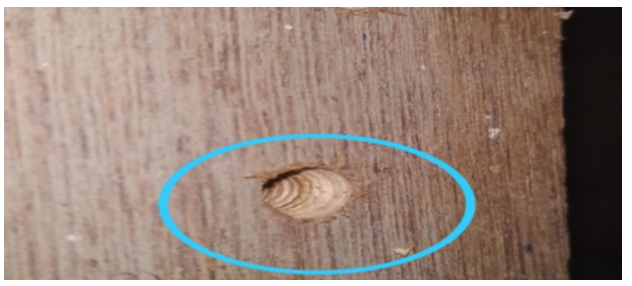

Figure 2. Output of Screw Threading

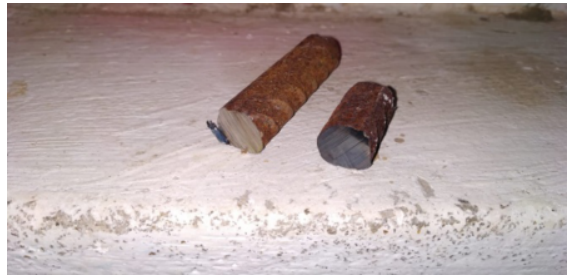

Figure 3. Output of Screw Threading

\section{Conclusion and Future Scope}

With appropriate measures this will fulfill our requirement partially. Sometimes there will be delay in this process and it will harm to the property and life, if any malfunction occurs on Motors or sudden High short circuit in power line which leads to fire. To overcome the existing drawback a new system is proposed for metal cutting and screw threading. Using IOT and Artificial Intelligence, intelligent decisions are made in the Industrial Automation.

\section{References}

[1] Sadeque Khan Professor Dr.M.S. Bhat .GUI Based Industrial Monitoring and Control System IEEEpaper, 2015.

[2] S.d.t. Kelly, n.k. Suryadevara and S.C. Mukhopadhyay Towards the Implementation of IOT for Environmental Condition Monitoring in Homes, IEEE

[3] Pooja Kanase1, Sneha Gaikwad2, Smart Hospitals Using Internet of Things (IOT), International Research Journal of Engineering and Technology (IRJET),2018.

[4] RajeevPiyare and Seong Ro Lee. Smart Home-Control and Monitoring System Using Smart Phone.ICCA 2015, ASTL Vol. 24, pp. 83 - 86, 2015 C SERSC.

[5] Gopinath Sundaram, BhanuprasadPatibandala, Harish Santhanam .Smart System using Internet of Things.in 2017.

[6] S L S Sri Harsha1, S Chakrapani Reddy.School of Computing, Sathyabama University, Chennai, Tamilnadu, India in 2016.

[7] Soumya S, Malini Chavali, Shuchi Gupta, Niharika Rao.Internet of Things based Home Automation System, IEEE International Conference on Recent Trends in Electronics Information Communication Technology, May 20-21, 2016, India,978-1-5090-0774-5/16/ C) 2016 IEEE 848.

[8] International conference on I-SMAC (IOT in Social, Mobile, Analytics and Cloud) I-SMAC2017 Enhanced Home Automation using IOT.

[9] A Hybrid Petri Net Approach for Polyethylene Terephthalate Bottle Manufacturing System B Muthuraj, V Mahesh, R Senthil - Journal of Computational and Theoretical Nanoscience, 2016.

[10] Simulation Of Three Tank System Using Hybrid Perti Net For Batch Process Dr. R.Senthil B.Muthuraj, Dr.V.Mahesh 2015/11/2 International Journal of Applied Engineering Research 47894798 Research India 